\title{
INTELIGENCIA EMOCIONAL Y AUTOESTIMA EN ESTUDIANTES DE LA CIUDAD DE LIMA METROPOLITANA CON Y SIN PARTICIPACION EN ACTOS VIOLENTOS
}

\author{
Carlos M. Velásquez Centeno ${ }^{1}$
}

El presente estudio, tiene como objetivo, establecer la relación existente entre la Inteligencia Emocional (IE) y la Autoestima (Aut), en una muestra intencional de 1014 escolares de educación secundaría de Lima Metropolitana, de ambos sexos, entre 11 a 19 años de edad, con $(n=510)$ y $\sin (n=504)$ participación en actos violentos, utilizando la Prueba de Inteligencia Emocional de Escurra-Aparcana-Ramos y la Escala de Autoestima de Coopersmith, quedando adaptada semántica y empíricamente.

Los Resultados arrojan una correlación entre moderada y alta, con una signo entre .01 y .05 en las Áreas de Autoconocimiento Emocional (AE), Control Emocional (CE), Automotivación $(A V)$, Empatía (EM), Habilidades para las Relaciones Interpersonales (RI) y la IE Total, lo cual nos permite medir la habilidad de percibir con precisión, estimar y expresar las emociones. Mientras que la relación entre la IE y la Aut se ubican en niveles bajos y muy bajos, con un nivel de sigo entre $.01 \mathrm{y} .05$, lo que nos demuestra cierta independencia entre estas variables.

El grupo de alumnos sin participación en actos violentos muestran una mayor IE y Aut que los violentos.

El grupo de mujeres presentan una mayor IE que el grupo de varones, salvo en AV donde el sexo no marca la diferencia en la motivación para el logro de una meta

En cuanto a la Aut general el grupo de varones muestra un mayor rango que las mujeres determinado esto por factores culturales.

PALABRAS CLAVES: Inteligencia Emocional, Autoestima, alumnos violentos, alumnos no violentos

The present research has as objective, establishment the relationship between Emocional Intelligence (El) and self-esteem (SE), in an intentional sample of 1014 secundary students from Lima Metropolitana, of both kinds, between 11 and 19 years old, with $(n=510)$ who participate in violent acts and (504) who don't participate in violent acts, using two instruments: Escurra-Aparcana-Ramos Emotional Intelligence Test and Coopersmith Selfesteem Scale, remaining semantic and empirical adapted.

The results yield between rnoderated and high correlation, with a significant between .01 and .05 in areas as Ernotional self Knowledge, Emotional Control, Selfmotivation, Empathy, Interpersonal relationship abilities and total Emotional Intelligence. These results let us measure the ability to perceive with precission, to estimate and to express emotions. While the relationship between Emotional Intelligence and Self-esteem are located in low and lowest standards with a significant standard between .01 and .05 demonstrating a bit independence between variables.

The group of students who don't participate in violent acts demonstrated a greater Emotional Intelligence and Self-esteem than the group of students who participate in violent acts.

\footnotetext{
${ }^{1}$ Miembros: García, Lupe; Montgomery, William; Guevara, Gilmar; Arenas, Carlos; García, Pedro y Gutiérrez, Víctor.

Colaboradores: Biminchumo, Liliana y Girau, Jean
} 
Girls presents a greater Emotional Intelligence than guys, except in Self-motivation where there are no difference by the kind in the motivation to get an objective. While in general Self-esteem, guys demonstrate a greater range than girls, determined by cultural factors.

KEY WORDS: Emotional intelligence, Self-esteem, violent scholars, no violent scholars.

\section{INTRODUCCIÓN}

Actualmente el mundo entero viene afrontando actos de violencia de todo tipo y por muy diversas causas. Nuestro país no se escapa de este fenómeno social el cual debe ser estudiado rigurosamente con el fin de comprenderlo y diseñar estrategias de afrontamiento que le permitan encararlo.

Sin duda alguna, el estudio de la violencia como fenómeno psicológico es muy complejo, de ahí la necesidad de descomponerlo para explicarlo; por esta razón es que en el presente estudio se abordarán dos variables psicológicas: la autoestima y la inteligencia emocional en el marco de la violencia escolar en Lima Metropolitana.

El caso de la autoestima, como vivencia psíquica es tan antigua como el ser humano, pero como constructo psicológico se remonta a James, W. (1890), quien estudia el desdoblamiento de nuestro yo - global en un yo conocedor y un yo-conocido, de este desdoblamiento nace la autoestima. En la actualidad un gran número de autores concluyen que existen cuatro formas de definir la autoestima:

- El primer tipo es el enfoque actitudinal, en este caso las definiciones se basan en la idea de que el self puede ser tratado como un objeto de atención, como cualquier otra cosa o posibilidad. Del mismo modo que tenemos reacciones cognitivas, emocionales y conductuales hacia otros objetos, también podemos tenerla hacia nuestro self.

- El segundo tipo de definición desarrollada por los científicos sociales también comprenden la autoestima en términos de actitud, pero de un modo más sofisticado. Se refieren a la autoestima como la relación entre diferentes muestras de actitudes. La diferencias entre las actitudes propias hacia los sueños y los logros, o a la diferencia entre el self real e ideal.

- La tercera forma de definir la autoestima se centra en las respuestas psicológicas que la persona sostiene de su self. Estas repuestas se describen normalmente como la naturaleza afectiva basada en el sentimiento, es decir, positivo versus negativo, o aceptación versus rechazo.

- La cuarta forma de definir la autoestima, según Well E. \& Marwell G. (1988), manifiestan que la autoestima puede verse como una función o componente de la personalidad. En este caso la autoestima se considera como parte de uno mismo o sistema del self, normalmente la vincula a la motivación y/o autorregulación.

Entre otras formas de definir la autoestima ubicamos a Smelser (1989), quien trata de definir los componentes del concepto, en vez de analizar los tipos, presentando tres aspectos a considerar:

- Existe un primer componente cognitivo, la autoestima implica caracterizar algunas partes del self en términos descriptivos como: poder, seguridad. Implica preguntar qué tipo de persona es uno.

- En segundo lugar, existe un elemento afectivo, una valencia o grado de naturaleza positiva o negativa adherida a estas facetas identificadas, llamamos a esto alta o baja 
autoestima.

- En tercer lugar, en relación con el segundo existe un elemento evaluador, la atribución de un valor sobre la base de algún modelo ideal.

Es decir algunas definiciones ven la autoestima como razonablemente estable a lo largo del tiempo. Otras se refieren a la autoestima como responsiva a las influencias situacionales y contextuales, lo que implica o fluctúa a lo largo del tiempo.

Toda la información existente parece relacionar la autoestima con la salud mental positiva y el bienestar psicológico. Como dicen Bednar, R, Wells, G. \& Peterson, S. (1989 p. 190), «se ha demostrado repetidas veces que la autoestima y la salud psicológica se relacionan con consecuencias psicológicas favorables en diversas situaciones psicológicas», tal como los refieren los estudios realizados por:

Coopersmith (197-8), quien encontró en sus investigaciones que sujetos con alta autoestima eran individuos activos, expresivos, tendían al éxito académico como social. También que son poco sensibles a la crítica y que los sentimientos de ansiedad los perturban en pequeña escala. Una autoestima positiva, permite conductas afectivas competentes, sentimiento de satisfacción interior y de aceptación. De la autoestima dependen las expectativas de éxito y la amplitud para enfrentar a la ansiedad.

Panizo, M. (1985) realizó una investigación en colegios de Lima, sobre autoestima y rendimiento escolar en una muestra de 305 niños y 397 niñas, tanto de colegios particulares como parroquiales. Encontró que existe asociación significativa entre la autoestima y el rendimiento escolar, los niños que presentan alta autoestima presentan alto rendimiento escolar. Así mismo, encontró diferencias en el nivel de autoestima con relación al sexo, los varones obtuvieron mejores puntuaciones que las mujeres tanto en el test Inventario de Coopersmith como en la escala de sí mismo.

Matos Ruth (1996). Trabajo con 108 alumnos de una Universidad Particular de Lima Metropolitana, procediendo a separarlos en dos grupos los que presentan problemas con su carrera y los que no. Aplicándoles el Inventario de Autoestima, el Inventario de Temperamento de Guilford Zimmerman y el Registro de Preferencias Vocacionales de Kuder. Los resultados obtenidos determinaron que si existe relación entre la autoestima, la personalidad y la elección vocacional en función a la ocupación de los padres y la condición socioeconómica.

Pecho, J. (1998), Trabajo con niños y adolescentes de entre 7 y 17 años que reciben apoyo no gubernamental los centros estudiantiles de Lima, donde se trabajó con un diseño descriptivo comparativo. Se procedió a la aplicación colectiva de pruebas (CAES, ZACK y CAP) a grupos de 5 a 15 menores.

Al término de la investigación se halló que no existen correlaciones significativas entre la autoestima y las habilidades sociales en total, aunque sí entre los sujetos del género masculino del mismo periodo cronológico.

Pequeña, J. (1999). El objetivo de su investigación fue determinar el grado de relación que existe entre la autoestima y el locus de control en estudiantes universitario de las distintas especialidades profesionales que cursaban el primer semestre académico de la UNMSM. La muestra fue de 403 casos seleccionados al azar. Utilizó la Escala de Autoestima de Rosemberg y la Escala de Locus de Control para operacionalizar los constructos de los participantes de la muestra. El planteamiento nace del supuesto de que la auto estima es el fenómeno que se traduce en conceptos, imágenes y juicios de valor referidos a sí mismo; es un proceso psicológico cuyos contenidos son socialmente determinados. La autoestima tiene mucha importancia en cada aspecto de nuestra vida. El Locus de Control está relacionado con la capacidad que tiene un sujeto para controlar el medio. En su resultado se observa que existe un alto grado de relación entre sus variables y 
por lo tanto se acepta su hipótesis alterna.

En cuanto al concepto de Inteligencia Emocional este se origina del concepto de inteligencia social que formula Thorndike (1920, Pp.228), quien la define como «la habilidad para comprender y dirigir a los hombres y mujeres, muchachos y muchachas $y$, actuar sabiamente en las relaciones humanas». Gardner H. (1983), revoluciona el concepto de inteligencia a través de la teoría de Inteligencias Múltiples, introduciendo dos tipos de inteligencias muy relacionadas con la competencia social: la inteligencia interpersonal y la inteligencia intrapersonal. Salovey P (1993 p.433) es quien acuña el concepto de Inteligencia Emocional como «un tipo de inteligencia social que incluye la habilidad de supervisar y entender las emociones propias y las de los demás, discriminar entre ellas y usar la información para guiar el pensamiento y las acciones de uno». Según Salovey \& Mayer (1990) el concepto de inteligencia emocional sub sume a los conceptos de inteligencia intrapersonal e interpersonal que propone Gardner, H. (1993), dándole además un enfoque algo menos cognitivo, pero añadiéndole el componente emocional, este concepto incluye además el hecho de que son metahabilidades que pueden ser categorizadas en cinco competencias o dimensiones, que se presume conforman su estructura conceptual y que involucra los siguientes aspectos:

- La evaluación y expresión de la emoción del yo, referido a la identificación y comprensión de las emociones en uno mismo, es decir la persona se percata tanto de su estado de ánimo como de su pensamiento que le da un mayor control sobre su vida.

- La evaluación y reconocimiento de las emociones en los demás, implica la habilidad para identificar exactamente las emociones de los demás, lo que parece estar relacionada con la empatía, la cual puede comprender tanto nuestra habilidad para identificar los sentimientos de los demás, así como el acceso general a nuestro propios sentimientos.

- La regulación de las emociones de si mismo y de los demás, referida a la dirección de las emociones de uno mismo, a la meta-experiencia del estado de ánimo, evaluando y actuando para cambiar nuestros estados de ánimo desagradables mientras mantenemos los agradables.

- El uso de la emoción para facilitar el rendimiento, que consiste en dirigir las emociones al servicio de un objetivo, siendo esencial para la atención selectiva, la automotivación y otros.

Para Goleman D. (1996), la inteligencia emocional, es un concepto amplio que incluye la habilidad para motivarse y persistir frente a las frustraciones, controlar impulsos y postergar gratificaciones, regular los estados de humor, evitar que las desgracias obstaculicen la habilidad de pensar, desarrollar empatía y optimismo, haciendo referencia a las siguientes habilidades:

- El conocimiento de las propias emociones, es decir, el conocimiento de uno mismo, la capacidad de reconocer un mismo sentimiento en el mismo momento en que aparece, constituyendo la piedra angular de la inteligencia emocional.

- La capacidad para controlar las emociones, la conciencia de uno mismo es una habilidad básica que nos permite controlar nuestros sentimientos y adecuarlos al momento.

- La capacidad de motivarse a sí mismo, esta habilidad ayuda a aumentar la competencia no sólo social sino también la sensación de eficacia en las empresas que se acometen.

- El reconocimiento de las emociones ajenas, la empatía, es decir la capacidad para poder sintonizarse con las señales sociales sutiles, que nos indican qué necesitan o 
qué quieren los demás.

- El control de las relaciones, es una habilidad que presume el relacionarnos adecuadamente con las emociones ajenas.

Por consiguiente la inteligencia emocional es concebida como sinónimo de carácter, personalidad o habilidades blandas, que se concretan en las cinco habilidades emocionales y sociales reseñadas y que tienen su traducción en conductas manifiestas, tanto en el ámbito de pensamiento, reacciones fisiológicas y conductas observables, aprendidas y aprensibles.

En nuestro medio Escurra L. y Cols (2000), construyeron una prueba para medir la Inteligencia Emocional tomando como base la propuesta de Solovey y Mayer. Construyeron items pertenecientes a los siguientes cinco dominios: a) Autoconocimiento Emocional, b) Control Emocional, e) Automotivación, d) Empatía y e) Habilidad para las relaciones Interpersonales. La prueba se aplicó a una muestra de estudiantes del primer semestre de las diversas especialidades de la UNMSM. Los cuales corresponden a una muestra aleatoria de 489 alumnos, pertenecientes de manera representativa a las 19 Facultades de las 5 áreas de especialización. El análisis Psicométrico de los items permitió eliminar aquellos que no discriminaban, la prueba presento Confiabilidad por consistencia interna, a través del coeficiente Alfa de Crombach tanto en general como en los dominios evaluados. También se observó que el instrumento presentó validez de contenido y validez de constructo.

En cuanto a los estudios sobre violencia en nuestro medio tratan de dar explicación al fenómeno psicosocial, entre las que tenemos:

Elizalde y cols (1997) en estudiante universitarios, Velásquez y Cols (1999) en estudiante de Lima Metropolitana, Velásquez y col s (2000) en estudiantes de secundaria de Lima y Trujillo. Así también encontramos en nuestro medio investigaciones cualitativas, Morales (1989), Tong (1998), Montgomery (1998), Martínez y Tong (1998), Cruzadi (1997), Arenas (1998), quienes describen, diagnostican y formulan soluciones de acuerdo a diferentes enfoques y criterios. Por otro lado la Psicología Comunitaria representada por Vallejo y Montero (1990), desarrollaron planes y aplicaciones prácticas al problema.

Por su parte el Congreso de la Republica ha creado varias Comisiones Técnicas y Parlamentarias que han tratado de conceptualizar el tema a la vez que han apoyado el trabajo readaptativo y preventivo del INABIF, la labor preventiva del PROMUDEH, así como también del programa de prevención de la violencia del Ministerio de Educación que se viene desarrollando en los Centros Educativos de mayor incidencia de actos violentos de Lima y algunas ciudades del interior del país.

Por otro lado se han desarrollado algunos estudios pilotos realizados por Anicama, J., Mayorga, R., Hinostroza, C., Antinori, B. Ochoa, M. (1997); Anicama, J., Vizcardo, I, Carrasco, J. Mayorga, E. (1999) y Varona (1991), han probado con éxito los efectos de programa de intervención sobre las habilidades sociales en alumnos con conductas agresivas.

Del mismo modo existen entidades internacionales como la OPS y nacionales como CEDRO que vienen realizando programas de prevención de la violencia intra y extra familiar, abordándola desde una metodología cualitativa.

Frente a todos los antecedentes mencionados consideramos importante conceptualizar la violencia como un problema de salud y desarrollo en el Perú que requieren de un abordaje inmediato con el fin de contribuir a su comprensión desde un punto de vista cuantitativo, lo que nos lleva a formular el siguiente problema: ¿Cuál es la relación entre la inteligencia emocional y la autoestima en estudiantes de secundaria de la ciudad de Lima Metropolitana con y sin participación en actos violentos? 


\section{MÉTODO}

\section{Muestra.}

La muestra intencional estratificada, estuvo conformada por 1014 alumnos de 22 Centros Educativos Nacionales de los diferentes Conos de Lima Metropolitana cuyas edades fluctuaron entre los 11 y 19 años, de los cuales 504 no participan en actos Violentos y 510 participaron en actos violentos, en cuanto al sexo 735 fueron varones y 279 mujeres.

\begin{tabular}{|c|c|c|c|c|c|c|c|}
\hline \multirow{2}{*}{ CONO } & \multirow{2}{*}{ CENTRO EDUCATIVO } & \multicolumn{2}{|c|}{ SEXO } & \multicolumn{2}{|c|}{ CONDICION } & \multirow[b]{2}{*}{$\mathrm{CONO}$} & \multirow{2}{*}{$\begin{array}{c}\text { TOTAL } \\
\text { GENERAL }\end{array}$} \\
\hline & & $M$ & $\mathrm{~F}$ & V V & $\mathrm{N} \mathrm{V}$ & & \\
\hline \multirow{5}{*}{ NORTE } & Estados U nidos & 29 & 12 & 18 & 23 & & 41 \\
\hline & José Granda & 22 & 21 & 22 & 21 & & 43 \\
\hline & Carlos Wiesse & 37 & .05 & 21 & 21 & 221 & 42 \\
\hline & Peruano Suizo & 28 & 17 & 24 & 21 & & 45 \\
\hline & José M. Arguedas & 35 & 15 & 28 & 22 & & 50 \\
\hline \multirow{5}{*}{ ESTE } & Ricardo Palma & 33 & 14 & 24 & 23 & & 47 \\
\hline & Manuel G. Prada & 33 & 17 & 25 & 25 & & 50 \\
\hline & Antenor Orrego & 25 & 20 & 24 & 21 & 242 & 45 \\
\hline & Nicolás Copérnico & 34 & 16 & 25 & 25 & & 50 \\
\hline & San Marcos & 37 & 13 & 25 & 25 & & 50 \\
\hline \multirow{5}{*}{ SUR } & José C. Mariátegui & 24 & 24 & 25 & 23 & & 48 \\
\hline & Inca Pachacutec & 28 & 19 & 25 & 22 & & 47 \\
\hline & Ramiro Priale & 23 & 20 & 19 & 24 & 222 & 43 \\
\hline & Ricardo Palma & 42 & 00 & 21 & 21 & & 42 \\
\hline & Peruano Suizo & 25 & 17 & 21 & 21 & & 42 \\
\hline \multirow{5}{*}{ CERCADO } & Guadalupe & 48 & 01 & 24 & 25 & & 49 \\
\hline & Melitón Carbajal & 40 & 00 & 20 & 20 & & 40 \\
\hline & Alfonso Ugarte & 45 & 00 & 24 & 21 & 230 & 45 \\
\hline & Mariano Melgar & 46 & 00 & 22 & 24 & & 46 \\
\hline & Pedro A. Labarte & 35 & 15 & 25 & 25 & & 50 \\
\hline \multirow{3}{*}{ CALLAO } & República de Venezuela & 28 & 21 & 23 & 26 & \multirow{2}{*}{99} & 49 \\
\hline & Dos de Mayo & 38 & 12 & 25 & 25 & & 50 \\
\hline & TOTAL & 735 & 279 & 510 & 504 & 1014 & 1014 \\
\hline
\end{tabular}

\section{Instrumentos.}

La medición de la Autoestima se realizo a través de la Escala de Autoestima de Coopersmith (SEI) versión escolar, la cual fue adaptada semántica y estadísticamente para nuestra realidad. La muestra estratificada estuvo constituida por 362 alumnos de los C.E. secundarios de los cinco conos de Lima Metropolitana. Al no obtenerse la Validez y Con fiabilidad del SEI, se procedió con el Análisis Factorial de la escala quedando finalmente reestructurada en dos factores Autoestima Sí Mismo (ASM) y Autoestima Social (AS) cumpliendo ambos con los criterios de validez y confiabilidad. (Anexo No 1 y 2).

La medición de la Inteligencia Emocional, se realiza a través de la Prueba de Inteligencia Emocional de Escurra, Aparcana y Ramos, la cual fue adaptada semántica y estadísticamente para nuestra realidad. Se utilizó una muestra estratificada de 573 alumnos de los C.E. secundarios de los cinco conos de Lima Metropolitana. Luego de la eliminación de algunos items que no se ajustaron a las áreas de la Prueba, se obtuvo la validez y confiabilidad, manteniendo su misma estructura. (Anexo No 3 y 4)

\section{Procedimiento.}

La selección de los CE en los cuales se tuvo reporte de la existencia de actos violentos se realizó en conjunto con la Coordinación del Programa de Prevención de Violencia de la 
Oficina de Tutoría y Prevención Integral del Ministerio de Educación. Luego se procedió a la realización de coordinaciones con los Directores de cada uno de los CE para la administración de los instrumentos psicológicos, quienes sobre la base del conocimiento de su realidad agruparon a los alumnos de secundaria en dos grupos de acuerdo a la variable participación o no participación en actos violentos.

Cuadro No 1: Prueba de Normalidad de Kolmogorov-Smirnov

\begin{tabular}{|ccccccccccc}
\hline AREAS & AE & CE & AU & EM & RI & IEG & ASM & AS & AUTG \\
\hline $\mathrm{N}$ & 1014 & 1014 & 1014 & 1014 & 1014 & 1014 & 987 & 999 & 915 \\
Parámetros normales ab Media & 209073 & 368264 & 585187 & 173343 & 307968 & 1643836 & 18192 & 7.5596 & 251344 \\
Desviación típica & 4.1625 & 6.6833 & 10.0100 & 3.8298 & 6.0529 & 255574 & 1.5291 & 1.6430 & 2.5299 \\
Diferencias más extremas absoluta & .068 & .049 & .054 & .073 & .049 & .033 & .169 & .157 & .106 \\
Positiva & .043 & .036 & .026 & .037 & -.025 & .018 & .116 & .092 & .065 \\
Negativa & -.068 & -.049 & -.054 & -.073 & -.049 & -.033 & -.169 & -.157 & -.106 \\
Z de Kolmgorow & 2.166 & 1.554 & 1.716 & 2.323 & 1.566 & 1.060 & 5321 & 4.069 & 3307 \\
Sig. Asintót. (bilateral) & .000 & .016 & .006 & .000 & .015 & 211 & .000 & .000 & .000 \\
\hline
\end{tabular}

a.b. La distribución de contraste es la Normal. Se ha calculado a partir de los datos

La verificación de la normalidad de las puntuaciones de las áreas de la Prueba de Inteligencia Emocional y de Autoestima, permitió el uso de una prueba no paramétrica como la U de Mann- Whitney

Cuadro No 2: Prueba de U de Mann- Whitney de la Pruebas de Inteligencia Emocional y Autoestima en estudiantes de educación secundaria de Lima Metropolitana con y sin participación en actos violentos

\begin{tabular}{|c|c|c|c|c|c|}
\hline \multirow{2}{*}{ Pruebas } & \multirow{2}{*}{ Áreas } & \multicolumn{2}{|c|}{ Rango de medias } & \multirow{2}{*}{$\mathrm{U}$} & \multirow{2}{*}{$\mathrm{P}$} \\
\cline { 3 - 4 } & No violentos & Violentos & & .000 \\
Inteligencia & $\mathrm{AE}$ & 540.89 & 474.50 & 111689.5 & .000 \\
emocional & $\mathrm{CE}$ & 569.54 & 451.13 & 99771.0 & .049 \\
& AU & 525.73 & 489.49 & 119334.0 & .000 \\
& $\mathrm{EM}$ & 561.06 & 454.57 & 101526.5 & .000 \\
\hline \multirow{4}{*}{ Autoestima } & RI & 555.56 & 460.00 & 104297.5 & .000 \\
& IEG & 556.87 & 458.71 & 103638.0 & .000 \\
& ASM & 535.34 & 454.87 & 101839.0 & .008 \\
& AUTG & 524.36 & 476.64 & 112781.5 & .000 \\
\hline
\end{tabular}

Se aprecia que de acuerdo a los resultados obtenidos en el Cuadro No 2, existen diferencias significativas en todas las áreas de la Prueba de Inteligencia Emocional y Autoestima donde los alumnos que No participan en actos violentos tienen predominio sobre los Violentos.

Cuadro $\mathbf{N}^{\circ}$ 3: Prueba de $\mathrm{U}$ de Mann-Whitney de las pruebas de Inteligencia emocional y Autoestima en estudiantes de educación secundaria de Lima Metropolitana por Sexo.

\begin{tabular}{|l|l|l|l|l|l|}
\hline Pruebas & \multirow{2}{*}{ Áreas } & \multicolumn{2}{|l|}{ Rango de medias } & U \\
\cline { 3 - 4 } & & Masculino & Femenino & & \\
\hline Inteligencia & AE & 495.83 & 538.26 & 93951.500 & .039 \\
emocional & CE & 492.80 & 546.23 & 917276.500 & .009 \\
& AU & 497.58 & 533.63 & 95241.000 & .080 \\
& EM & 497.15 & 587.45 & 80255.500 & .000 \\
& RI & 490.73 & 551.68 & 90206.000 & .003 \\
& IEG & 489.03 & 556.15 & 88959.000 & .001 \\
\hline Autoestima & IEG & 501.76 & 473.71 & 91921.500 & .158 \\
& ASM & 508.92 & 476.29 & 92626.500 & .105 \\
& AUTG & 499.99 & 456.20 & 86028.500 & .029 \\
\hline
\end{tabular}

Como se observa en el Cuadro No 3, existen diferencias significativas en las áreas de 
Autoconocimiento Emocional, Control Emocional, Empatía, Habilidades para las Relaciones Interpersonales e Inteligencia Emocional General siendo esta mayor en las mujeres, mientras que no existen diferencias en el área de Automotivación de la Prueba de Inteligencia Emocional.

En cuanto a las áreas de la Autoestima Si Mismo y Autoestima Social no existen diferencias, pero en cuanto a la Autoestima Total se encontraron diferencias siendo mayor en los Varones.

\section{Cuadro No 4: Matriz de Correlación General entre la Inteligencia Emocional y la Autoestima en estudiantes de educación secundaria con y sin participación en actos violentos.}

\begin{tabular}{|l|l|l|l|l|l|l|l|l|}
\hline & CE & AU & EM & RI & IEG & ASM & AS & AUTG \\
\hline AE & .538 & .563 & .432 & .558 & .721 & .153 & .202 & .221 \\
CE & & .708 & .550 & .637 & .859 & .225 & .259 & .372 \\
AU & & & .555 & .676 & .912 & .288 & .309 & .301 \\
EM & & & .477 & .694 & .033 & .164 & .128 \\
RI & & & & .830 & .203 & .281 & .303 \\
IEG & & & & & & .249 & .312 & .351 \\
ASM & & & & & & & .268 & .780 \\
AS & & & & & & & & .812 \\
\hline
\end{tabular}

El análisis de la matriz de las correlaciones entre las áreas de la Prueba de Inteligencia Emocional en estudiantes de educación secundaria con y sin participación en actos violentos (Cuadro No 4), permite observar que las correlaciones en cada una de las áreas son positivas, estadísticamente significativas y con una $\mathrm{r}$ entre .477 y .708 . En cuanto a la correlación entre las áreas de la prueba de inteligencia emocional con la inteligencia emocional general esta es positiva, estadísticamente significativa y una $r$ entre $.694 \mathrm{y}$ .912. La correlación entre las áreas de la prueba de inteligencia emocional y las de autoestima son positivas y con un $\mathrm{r}$ entre .203 y .309. En cuanto a la correlación entre las áreas de la prueba de autoestima con la autoestima general estas son positivas, estadísticamente significativas y con una $\mathrm{r}$ entre .780 y .812

\section{Cuadro No 5: Matriz de Correlación entre la Inteligencia Emocional y la Autoestima en estudiantes de educación secundaria con participación en actos violentos}

\begin{tabular}{|l|l|l|l|l|l|l|l|l|}
\hline & CE & AU & EM & RI & IEG & ASM & AS & AUTG \\
\hline AE & .534 & .569 & .453 & .562 & .723 & .099 & .205 & .194 \\
CE & & .711 & .557 & .634 & .858 & .143 & .273 & .265 \\
AU & & & .583 & .661 & .917 & .206 & .307 & .327 \\
EM & & & & .464 & .705 & .028 & .178 & .094 \\
RI & & & & & .819 & .134 & .224 & .228 \\
IEG & & & & & & .163 & .305 & .298 \\
ASM & & & & & & .791 \\
AS & & & & & & \\
\hline \multicolumn{7}{|c|}{$\mathrm{N}=510 \quad \mathrm{p}=.01$} \\
\hline
\end{tabular}

Al analizar la matriz de las correlaciones entre las áreas de la Prueba de Inteligencia Emocional en estudiantes de educación secundaria con participación en actos violentos (Cuadro No.5), permite observar que las correlaciones de las áreas de la Prueba de Inteligencia Emocional son positivas, estadísticamente significativas y con una $\mathrm{r}$ entre .464 y.711.

En cuanto a la correlación entre las áreas de la Prueba de Inteligencia Emocional con la Inteligencia Emocional General esta son positivas, estadísticamente significativas y con una 
r entre .705 y .917.

Las Correlaciones entre las áreas de la Prueba de Inteligencia Emocional y las de Autoestima son positivas en su mayoría, estadísticamente significativa y con una $r$ entre .134 y .307, a excepción de la correlación entre EM y ASM que es negativa, no significativa con una $\mathrm{r}$ de .028.

En cuanto a la correlación entre las áreas de la Prueba de Autoestima con la Autoestima General esta son positivas, estadísticamente significativas y con una $\mathrm{r}$ entre. 782 y .791.

\section{Cuadro $\mathrm{N}^{\circ}$ 6. Matriz de correlación entre la inteligencia emocional y la autoestima en estudiantes de educación secundaria sin participación en actos de violencia}

\begin{tabular}{|l|l|l|l|l|l|l|l|l|}
\hline & CE & AU & EM & RI & IEG & ASM & AS & AUTG \\
\hline AE & .524 & .554 & .388 & .538 & .710 & .188 & .187 &. .227 \\
CE & & .713 & .507 & .614 & .853 & .280 & .230 & .308 \\
AU & & & .523 & .697 & .915 & .380 & .310 & .419 \\
EM & & & & .455 & .663 & .054 & .131 & .123 \\
RI & & & & & .833 & .246 & .320 & .352 \\
IEG & & & & & & .319 & .309 & .384 \\
ASM & & & & & & .290 & .766 \\
AS & & & & & & \\
\hline
\end{tabular}

La matriz de correlación entre las áreas de la Prueba de Inteligencia Emocional en estudiantes de educación secundaria sin participación en actos violentos (Cuadro No 6), permite observar que las correlaciones de las áreas de la Prueba de Inteligencia Emocional son positivas, estadísticamente significativas y con una r entre .388 y .713.

En cuanto a la correlación entre las áreas de la Prueba de Inteligencia Emocional con la Inteligencia Emocional General esta son positivas, estadísticamente significativas y con una r entre .663 y .915 .

Las Correlaciones entre las áreas de la Prueba de Inteligencia Emocional y las de Autoestima son positivas, estadísticamente significativas y con una r entre .054 y .320. En cuanto a la correlación entre las áreas de la Prueba de Autoestima con la Autoestima General esta son positivas, estadísticamente significativas y con una r entre .766 y .838 .

\section{DISCUSIÓN}

Existen diferencias significativas entre los grupos de alumnos que participan y No participan en actos violentos, siendo favorecidos a estos últimos en las Pruebas de inteligencia Emocional y Autoestima, tanto en cada una de sus áreas como en General (Cuadro No 2), esto indica que los alumnos que no participan en actos violentos son emocional mente más competentes, valoran sus cualidades y talentos en contraste con los que sí participan.

Las diferencias en cuanto al género en la Prueba de inteligencia Emocional, favorecen a las mujeres en las áreas de Autoconocimiento Emocional, Control Emocional, Empatía y Habilidades para las Relaciones Interpersonales (Cuadro No 3), determinando que las mujeres a diferencia de los varones logran discriminar, supervisar, comprender sus emociones y las de los demás, usando la información para guiar sus pensamientos y acciones. Mientras que en el área de Automotivación, el género no determina diferencias, por lo que tanto los varones como las mujeres se motivan así mismo y encausan sus emociones en el logro de sus metas. En cuanto a las áreas de Sí Mismo y Social de la Prueba de Autoestima, estas no se ven influenciadas por el género, sin embargo en la Autoestima General, esta es mayor en los varones que en las mujeres, coincidiendo con el estudio de Panizo (1985). 
En los grupos de los alumnos con y sin participación en actos violentos, encontramos relación entre la Prueba de Inteligencia Emocional y la de Autoestima, las cuales son positivas y significativas al .01 (Cuadro No 4). Destacando la relación entre el CE y la AU, es decir a mayor motivación de meta mayor es el control de sus sentimientos. Por otro lado en la Prueba de Autoestima, la relación entre la Autoestima General con sus áreas, fluctúan entre altas y muy altas. Por los resultados encontrados podemos conceptuar a la Inteligencia Emocional como la habilidad para motivarse, persistir frente a las frustraciones, controlar impulsos, regular los estados de humor, evitar que las desgracias obstaculicen las habilidades para pensar, desarrollar empatía y optimismo en la consecución de alguna meta (Goleman, D. 1996).

Así mismo observamos relaciones altas entre la Autoestima General y sus áreas: Si Mismo y Social, lo que nos permite alcanzar estimaciones más exactas del constructo «Autoestima».

Al relacionar las áreas y el puntaje General de las Pruebas de Inteligencia General y la de Autoestima, estas son bajas, debido probablemente a que la autoestima es responsiva a las influencias situacionales y contextuales, mientras que la Inteligencia Emocional es la capacidad que permite guiar el pensamiento y las acciones de uno para adecuarse eficazmente a su entorno.

La relaciones de los estudiantes con y sin participación en actos violentos por separado (Cuadros No 5 y 6), expresan los mismos niveles de asociación que la encontrada cuando se analizaron en conjunto (Cuadro N03).

Los resultados observados en cuanto al proceso de adaptación de la Escala de Autoestima de Coopersmith, nos permitió reestructurarla a través del análisis factorial, quedando finalmente dos áreas: Si Mismo y Social.

El proceso de adaptación de la Prueba de Inteligencia Emocional, permitió mantener su estructura, eliminando algunos items que en sus respectivas áreas no cumplieron con los criterios empíricos.

\section{CONCLUSIONES}

Los resultados del estudio nos permiten afirmar que:

- Existe una relación baja entre la Autoestima y la Inteligencia Emocional en estudiantes con y sin participación en actos violentos de Lima Metropolitana. La misma relación se expresa en los estudiantes que participan y no participan en actos violentos por separado.

- Los estudiantes que no participan en actos violentos presentan una mayor autoestima e inteligencia emocional que los que si participan.

- Las mujeres presentan un mejor autoconocimiento emocional, control emocional, empatía y habilidades para las relaciones interpersonales que los varones. La automotivación no se ve influenciada por él género.

- La prueba de autoestima queda reestructurada en dos áreas: Si Mismo y Social, para estudiantes de secundaria de Lima Metropolitana.

- La prueba de inteligencia emocional logra evaluar cinco áreas: autoconocimiento emocional, control emocional, automotivación, empatía y habilidades para las relaciones interpersonales en estudiantes de educación secundaria de Lima Metropolitana 


\section{REFERENCIAS BIBLIOGRÁFICAS}

Anicama, J., Mayorga, R., Hinostroza, C., Antinori, B. Ochoa, M. y Tomás, A. (1997). Efectos de un programa cognitivo-conductual para prevenir y controlar la violencia de menores en extrema pobreza y alto riesgo. Wiñay Yachay, Revista de la Facultad de Psicología UNFY. 2(1), 71-8I.

Anicama, J., Vizcardo, J., Carrasco, J. y Mayorga, E. (1999). Estudio epidemiológico sobre la violencia y comportamientos asociados en Lima Metropolitana y Callao. Lima, Perú: MINSA-UNFY.

Arenas, C. (1998). Los rostros escondidos y la acción social frente a la marginalidad: violencia juvenil de los 90. Revista de psicología de la UNMSM. 11 (1), 93-106.

Bednar, R., Wells, G. \& Peterson, S. (1989). Self-esteem: Paradoxes and innovations in clinical theory and practice. Washington D. c.: APA

Comisión Especial del Congreso de la República que investiga las causas y consecuencias de la violencia cotidiana en el país (1998). Iniciativa legislativa. Congreso de la República

Coopersmith, S. (1978) Estudios sobre la estimación propia en Psicología Contemporánea. Madrid: Blume.

Escurra L. y Cols (2000) Construcción de una prueba de inteligencia emocional. Revista de Investigación en Psicología de la UNMSM. Vol. 3, N 1, pp. 7185.

Elizalde, R.; Bulnes, M. Escurra, M. y Dávila, N. (1997). Actitudes hacia la violencia en jóvenes universitarios. Revista de psicología de la UNMSM. I (1),107-128.

Golemam D. (1996) Inteligencia Emocional. Barcelona. Kairos.

Gardner H. (1993) Inteligencias Múltiples: la teoría en la práctica. Barcelona. Paidos. Iberica

James, W. (1890) The principIes of psychology. Cambridge, Ma: Harvard University Press.

Panizo, M. (1985) Autoestima y rendimiento escolar en un grupo de niños de quinto grado. Tesis de Bachiller. Lima PUCP.

Pecho, J. (1996) Habilidades Sociales, Autoestima y percepción del Autoritarismo paterno en función del sexo y el periodo cronológico en niños y adolescentes de condición socioeconómica baja que reciben apoyo no gubernamental. Tesis de Licenciatura. Lima. UNMSM.

Pequeña, 1. (1999) Relación entre la autoestima y el locus de control en estudiantes del primer semestre de la UNMSM. Tesis de Magíster en Psicología. Lima. UNMSM.

Salovey, P \& Mayer, J.D. (1990) Emotional intelligence. Imagination, Cognition and Personality, 9,185-211

Smelser, NJ. (1989). Self-esteem and social problems: An introduction. In A. M. Mecca, N. J. Smelser, \& J. Vasconcellos (Eds.), The social importance of selfesteem. Berkeley: University of California Press.

Tong, F. (1995). Los jóvenes pandilleros. Entre el estigma y la epopeya. Lima: Tarea.

Vallejos, J. y Montero, V. (1990). La Psicología comunitaria en el Perú. Psicología actual. III (6), 15-2I.

Varona, (1991). Efectos de un programa para la sustitución de la agresión mediante el desarrollo de habilidades sociales y el autocontrol en menores de II - 15 años, en condiciones especialmente difíciles, institucionalizados del COMA IN. Tesis de Maestría, Lima:

UPCH.

Velásquez, C. (1999). La violencia juvenil en Lima Metropolitana. Revista de Investigación en Psicología de la UNMSM. Vol. 1, W 1, pp. 91-102. 
Velásquez, C. (2000). Ansiedad y Cólera en estudiantes de las ciudades de Lima y Trujillo con participación y no participación en actos violentos. Revista de Investigación en Psicología de la UNMSM. Vol. 3, W 1, pp. 39 - 54.

Well, E. \& Marwell G. (1988) Self-esteem: Its conceptualization and measure ment. Beverly Hills, CA: Sage. 


\section{ANEXOS}

Anexo No. 1

ANÁLISIS DE ITEMS DEL COEFICIENTE DE VALIDEZ ITEM - TEST CORREGIDO DE CADA UNA DE LAS ÁREAS DE LA PRUEBA DE AUTOESTIMA

\begin{tabular}{crcc}
\multicolumn{4}{c}{ AUTOESTIMA SI MISMO } \\
\hline ITEMS & $\mathrm{r}$ & ITEMS & $\mathrm{r}$ \\
\hline 10 & .3365 & 27 & .3327 \\
13 & .5336 & 29 & .4557 \\
15 & .4995 & 33 & .2505 \\
16 & .5329 & 34 & .2984 \\
17 & .3783 & 39 & .4426 \\
20 & .4701 & 44 & .3709 \\
22 & .4056 & 51 & .4226 \\
23 & .3779 & 52 & .3081 \\
24 & .5455 & 54 & .2514 \\
25 & .4297 & 55 & .2344 \\
\hline
\end{tabular}

\begin{tabular}{cc}
\multicolumn{2}{c}{ AUTOESTIMA SOCIAL } \\
\hline ITEMS & $\mathrm{r}$ \\
\hline 2 & .3172 \\
4 & .3789 \\
5 & .2268 \\
8 & .2616 \\
12 & .2181 \\
19 & .3651 \\
28 & .2388 \\
42 & .2356 \\
47 & .2679 \\
56 & .2269 \\
\hline
\end{tabular}

Anexo No. 2

CONFIABILIDAD DE LA PRUEBA DE AUTOESTIMA POR CONSISTENCIA INTERNA A TRAVES DEL COEFICIENTE ALFA DE CROMBACH

\begin{tabular}{|l|l|}
\hline Áreas & Coeficiente de correlación \\
\hline Autoestima Si Mismo & .8238 \\
Autoestima Social & .5943 \\
\hline
\end{tabular}


Anexo No.3

ANALISIS DE ÍTEMS DEL COEFICIENTE DE VALIDEZ ITEM-TEST CORREGIDO DE CADA UNA DE LAS ÁREAS DE INTELIGENCIA EMOCIONAL

\begin{tabular}{l|l}
\hline $\mathrm{AE}$ & $\mathrm{r}$ \\
\hline 01 & .3218 \\
02 & .4409 \\
03 & .4050 \\
06 & .3241 \\
79 & .2991 \\
106 & .2603 \\
& \\
& \\
& \\
& \\
& \\
& \\
& \\
& \\
\hline $\mathrm{RI}$ & $\mathrm{r}$ \\
\hline 07 & .3194 \\
09 & .4089 \\
26 & .3437 \\
38 & .2927 \\
48 & .2935 \\
70 & .3059 \\
103 & .3548 \\
104 & .3038 \\
105 & .4021 \\
\hline & \\
& \\
& \\
& \\
& \\
& \\
& \\
& \\
& \\
& \\
& \\
& \\
& \\
& \\
& \\
& \\
& \\
& \\
& \\
& \\
& \\
& \\
& \\
& \\
& \\
& \\
& \\
& \\
& \\
& \\
& \\
&
\end{tabular}

\begin{tabular}{l|l}
\hline CE & r \\
\hline 20 & .3119 \\
21 & .2914 \\
31 & .2993 \\
32 & .3244 \\
33 & .2801 \\
36 & .2275 \\
45 & .2446 \\
57 & .3451 \\
52 & .3288 \\
72 & .2163 \\
84 & .2878 \\
& \\
& \\
& \\
& \\
\hline EM & r \\
\hline 11 & .3561 \\
18 & .3482 \\
49 & .3836 \\
50 & .4184 \\
51 & .3108 \\
& \\
\hline
\end{tabular}

\begin{tabular}{l|l}
\hline AU & $\mathrm{r}$ \\
\hline 19 & .4095 \\
23 & .3864 \\
24 & .3708 \\
25 & .4096 \\
27 & .3833 \\
30 & .4162 \\
39 & .3809 \\
40 & .2817 \\
46 & .3244 \\
55 & .4155 \\
56 & .4502 \\
57 & .3444 \\
59 & .3809 \\
60 & .4880 \\
64 & .2810 \\
77 & .3938 \\
82 & .3427 \\
\hline & \\
\hline & \\
\hline
\end{tabular}

Anexo No. 4

CONFIABILIDAD DE LA PRUEBA DE INTELIGENCIA EMOCIONAL POR CONSISTENCIA INTERNA A TRAVÉS DEL COEFICIENTE ALFA DE CROMBACH

\begin{tabular}{|l|l|}
\hline Áreas & Coeficiente de correlación \\
\hline Autoconocimiento emocional (AE) & .6054 \\
Control emocional (CE) & .6322 \\
Automotivación (AU) & .7915 \\
Empatía (EM) & .6065 \\
Hab. Para relaciones interpersonales (RI) & .6591 \\
\hline
\end{tabular}

